\title{
Leptin reverses weight loss-induced changes in regional neural activity responses to visual food stimuli
}

\author{
Michael Rosenbaum, ${ }^{1}$ Melissa Sy, ${ }^{2}$ Katherine Pavlovich, ${ }^{1}$ Rudolph L. Leibel, ${ }^{1}$ and Joy Hirsch ${ }^{2}$ \\ 1Department of Pediatrics, Division of Molecular Genetics, Naomi Berrie Diabetes Center, and 2Departments of Radiology, Neuroscience, and Psychology, \\ fMRI Research Center, Columbia University Medical Center/New York Presbyterian Medical Center, New York, New York, USA.
}

\begin{abstract}
Increased hunger and food intake during attempts to maintain weight loss are a critical problem in clinical management of obesity. To determine whether reduced body weight maintenance is accompanied by leptinsensitive changes in neural activity in brain regions affecting regulatory and hedonic aspects of energy homeostasis, we examined brain region-specific neural activity elicited by food-related visual cues using functional MRI in 6 inpatient obese subjects. Subjects were assessed at their usual weight and, following stabilization at a $10 \%$ reduced body weight, while receiving either twice daily subcutaneous injections of leptin or placebo. Following weight loss, there were predictable changes in neural activity, many of which were reversed by leptin, in brain areas known to be involved in the regulatory, emotional, and cognitive control of food intake. Specifically, following weight loss there were leptin-reversible increases in neural activity in response to visual food cues in the brainstem, culmen, parahippocampal gyrus, inferior and middle frontal gyri, middle temporal gyrus, and lingual gyrus. There were also leptin-reversible decreases in activity in response to food cues in the hypothalamus, cingulate gyrus, and middle frontal gyrus. These data are consistent with a model of the weightreduced state as one of relative leptin deficiency.
\end{abstract}

\section{Introduction}

Over $60 \%$ of U.S. adults are overweight $\left(\mathrm{BMI}>25 \mathrm{~kg} / \mathrm{m}^{2}\right)$ or obese $\left(\mathrm{BMI}>30 \mathrm{~kg} / \mathrm{m}^{2}\right)$ and at risk for adiposity-related morbidities such as diabetes mellitus and hyperlipidemia (1). The intractable nature of obesity is reflected in the $75 \%-95 \%$ recidivism rate to obesity among the formerly obese (2). Maintenance of a reduced body weight, with resultant declines in circulating concentrations of leptin, is accompanied by decreased energy expenditure and increased hunger/food intake that act coordinately to favor the regain of lost weight in humans and rodents (3-8). The behavioral and metabolic changes characterizing individuals maintaining a $10 \%$ reduced body weight are largely reversed by the administration of "replacement" doses of leptin (6) that restore circulating leptin concentrations to pre-weight loss levels. The weight-reduced state may, therefore, be regarded as a condition of relative leptin insufficiency $(6,7)$.

Brain regions known to be involved in the regulation of energy intake have been identified largely through physical and, more recently, molecular lesioning of relevant CNS pathways (9). For example, the hypothalamus has afferent and efferent connections to the brainstem, midbrain, amygdala, hippocampus, and cortex, which, if selectively lesioned or stimulated, result in predictable alterations in feeding behavior (10).

In this study, blood oxygen level-dependent (BOLD) signals elicited by viewing food items during functional MRI (fMRI) were acquired to assess the effects of maintenance of a reduced body weight and replacement of exogenous leptin on neural activity

Nonstandard abbreviations used: BOLD, blood oxygen level-dependent; AMRI,

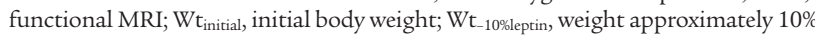
below $\mathrm{Wt}_{\text {initial }}$ with twice daily injections of recombinant methionyl human leptin;

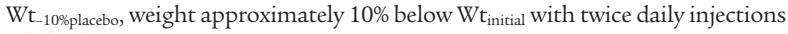
of placebo.

Conflict of interest: The authors have declared that no conflict of interest exists. Citation for this article: J. Clin. Invest. 118:2583-2591 (2008). doi:10.1172/JCI35055. relevant to energy intake in weight-reduced individuals. Neural responsiveness to visual food versus non-food cues was compared in 6 obese subjects while maintaining their initial body weight $\left(\mathrm{Wt}_{\text {initial }}\right)$ and after being stabilized at a weight approximately $10 \%$ below $\mathrm{Wt}_{\text {initial }}$ and then receiving twice daily injections of either placebo ( $\mathrm{Wt}_{-10 \% \text { placebo }}$ ) or recombinant methionyl human leptin in doses calculated to restore 8 am circulating leptin concentrations to those present at $\mathrm{Wt}_{\text {initial }}\left(\mathrm{Wt}_{-10 \% \text { leptin }}\right)(6)$. This was a singleblind crossover study design in which subjects served as their own controls. Subjects were inpatients being fed a liquid formula diet throughout the study (see Methods, Figure 1, and Table 1). We hypothesized that maintenance of a reduced body weight leads to leptin-mediated changes in areas of the brain known to mediate regulatory, emotional, and cognitive aspects of feeding behavior. We found that maintenance of a reduced body weight was associated with predictable changes in neural activity in these regions, many of which were reversed by leptin.

\section{Results}

All analyses compared BOLD signals as indications of neural activity elicited during passive exposure to food versus non-food cues. Data were analyzed in subjects prior to weight loss $\left(\mathrm{Wt}_{\text {initial }}\right)$ and following weight loss ( $\mathrm{Wt}_{-10 \% \text { placebo }}$ or $\left.\mathrm{Wt}_{-10 \% \text { leptin }}\right)$. In this design, subjects receiving leptin remained at reduced weight, thus permitting analyses of whether leptin administration to weight-reduced subjects reverses the effects of weight loss per se on neural activity relevant to energy intake. As detailed in Methods, identification of brain areas responsive to food versus non-food cues was made after correction for multiple comparisons with $P<0.005$. Comparisons between group pairs ( $\mathrm{Wt}_{\text {initial }} \mathrm{vs}$. $\mathrm{Wt}_{-10 \% \text { placebo }}$ to identify weight loss effects and $\mathrm{Wt}_{-10 \% \text { placebo }} \mathrm{vs}$. Wt $\mathrm{Wt}_{-10 \% \text { leptin }}$ to identify leptin effects) were made with significance prospectively defined as $P<0.05$. Since statistical thresholds (based on signal to nose) may 


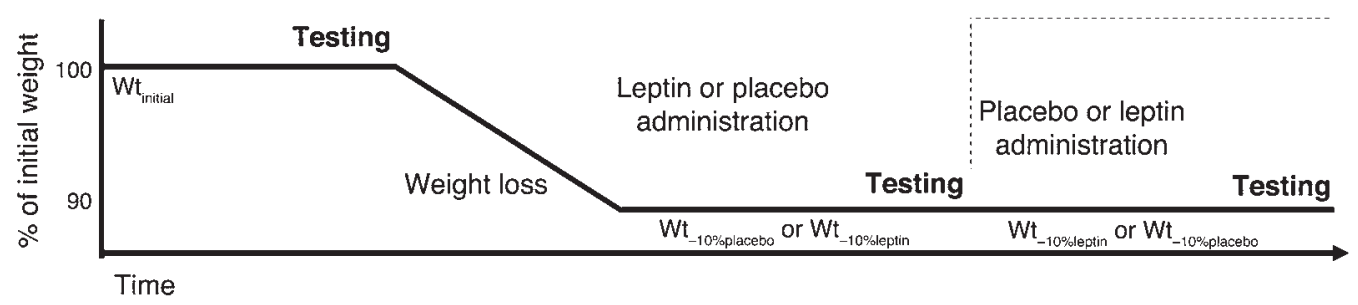

Figure 1

Schematic of protocol for 3 testing periods.

not be the best predictors of physiological significance, we have chosen the more complete report without further correction for multiple comparisons. The terms greater response and greater activity refer to a statistical comparison of BOLD signal amplitudes in specific brain areas that are greater in one condition relative to another at $P<0.05$. The precise $P$ values of significant betweengroup comparisons are reflected in the $\mathrm{z}$ scores for such comparisons that are given in Tables 2 and 3 .

Neural activity was greater at $W t_{-10 \% \text { placebo }}$ than at $W t_{\text {initial }}$. Brain areas in which neural activity was greater at $\mathrm{Wt}_{-10 \% \text { placebo }}$ than at $\mathrm{Wt}_{\text {initial }}$ represented a "long-range pattern" (i.e., a group of coactive but noncontiguous regions of the brain that are engaged during a specific task, indicative of a global pattern of brain activity that includes multiple regions) of food-related neural responses that were increased during maintenance of a reduced body weight. These areas included the limbic system (brainstem, parahippocampal gyrus, culmen, and globus pallidus) as well as systems mediating aspects of executive and decision-making functions (middle temporal gyrus, inferior frontal gyrus, middle frontal gyrus, and lingual gyrus) (Figure 2A and Table 2).

Neural activity was greater at $W t_{\text {initial }}$ than at $W t_{-10 \% p l a c e b o}$. Brain areas in which neural activity was greater at $\mathrm{Wt}_{\text {initial }}$ than at $\mathrm{Wt}_{-10 \% \text { placebo }}$ represented a long-range pattern of food-related neural responses that were more responsive during the maintenance of the initial weight (prior to weight loss) than during the maintenance of a reduced body weight (after weight loss). These areas included regions associated with metabolic, autonomic, and neuroendocrine aspects of energy homeostasis (hypothalamus), emotional control of food intake (amygdala, parahippocampal gyrus, and cingulate gyrus), integrative cognitive control functions (hippocampus, middle frontal gyrus, inferior parietal lobule, fusiform gyrus, and supramarginal gyrus), and motor planning (precentral gyrus) (Figure 2B and Table 2).
Neural activity was greater at $W t_{-10 \% \text { leptin }}$ than at Wt-10\%placebo. Brain areas in which neural activity was greater at $\mathrm{Wt}_{-10 \% \text { leptin }}$ than at $\mathrm{Wt}_{-10 \% \text { placebo }}$ represented a pattern of food-related neural processes that were more responsive during restoration of circulating leptin concentrations in subjects maintaining a reduced body weight. These areas included those associated with metabolic, autonomic, and neuroendocrine aspects of energy homeostasis (hypothalamus), error correction and performance monitoring (cingulate gyrus), decision making based on emotional and integrative control of food intake (inferior and middle frontal gyri, lingual gyrus, medial frontal gyrus, middle temporal gyrus, and precuneus), as well as limbic (culmen, putamen, and thalamus) and sensory (postcentral gyrus) regions (Figure $2 \mathrm{C}$ and Table 3 ). As hypothesized, the pattern of neural activity following leptin replacement during weight reduction was similar to the pattern observed when subjects were at $\mathrm{Wt}_{\text {initial }}$ (see Figure $2 \mathrm{~B}$ and Table 2). These common regions included hypothalamus, cingulate gyrus, and middle frontal gyrus.

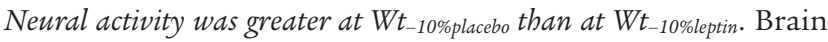
regions in which neural activity was more responsive during $\mathrm{Wt}_{-10 \% \text { placebo }}$ compared with $\mathrm{Wt}_{-10 \% \text { leptin }}$ represented a pattern of food-related neural processes that were more responsive during the maintenance of a reduced body weight (with the associated decline in circulating and CNS leptin concentrations) than at the same reduced weight but in a euleptinemic state. These areas included the limbic system (brainstem, parahippocampal gyrus, and culmen) as well as systems regulating executive and decision-making functions (middle temporal gyrus, inferior frontal gyrus, and middle frontal gyrus). Additionally, greater activity was observed in brain areas involved in enteric regulation (insula), cognitive control functions including error monitoring (cingulate gyrus), as well as knowledge representation (superior temporal gyrus and

Table 1

Subject characteristics

\begin{tabular}{|c|c|c|c|c|c|c|c|}
\hline Subject number & 1 & 2 & 3 & 4 & 5 & 6 & Mean \pm SEM \\
\hline Sex & Male & Male & Female & Female & Female & Female & \\
\hline Age & 44 & 43 & 35 & 40 & 36 & 33 & $38 \pm 2$ \\
\hline Height (cm) & 170 & 177 & 163 & 172 & 162 & 157 & $167 \pm 3$ \\
\hline \multicolumn{8}{|l|}{ Weight (kg) } \\
\hline $\mathrm{Wt}_{\text {initial }}$ & 138.5 & 117.4 & 80.5 & 176.2 & 102.1 & 94.5 & $118.2 \pm 14.6$ \\
\hline $\mathrm{Wt}_{-10 \% \text { placebo }}$ & 116.8 & 102.7 & 72.0 & 148.4 & 86.4 & 77.6 & $100.7 \pm 11.7^{\mathrm{A}}$ \\
\hline $\mathrm{Wt}_{-10 \% \text { leptin }}$ & 118.2 & 101.8 & 71.0 & 143.4 & 85.5 & 75.4 & $99.2 \pm 11.4^{A}$ \\
\hline $\mathrm{BMI}$ at $\mathrm{Wt}_{\text {initial }}\left(\mathrm{kg} / \mathrm{m}^{2}\right)$ & 47.9 & 37.5 & 30.3 & 59.6 & 38.9 & 38.3 & $42.1 \pm 4.2$ \\
\hline Testing order at reduced weight & Lep/Plac & Plac/Lep & Plac/Lep & Plac/Lep & Lep/Plac & Lep/Plac & \\
\hline
\end{tabular}

AP $<0.01$ compared with $\mathrm{Wt}_{\text {initial. }}$. Lep, leptin administration; Plac, placebo administration. 
Table 2

Effects of weight loss

\begin{tabular}{|c|c|c|c|c|c|c|}
\hline \multirow[t]{2}{*}{ Region } & \multirow[t]{2}{*}{ BA } & \multicolumn{3}{|c|}{ Coordinates } & \multirow{2}{*}{$\begin{array}{c}\text { z } \\
\text { score }\end{array}$} & \multirow{2}{*}{$\begin{array}{c}\text { Voxels } \\
(n)\end{array}$} \\
\hline & & $x$ & $y$ & $z$ & & \\
\hline \multicolumn{7}{|l|}{$\mathrm{Wt}_{-10 \% \text { placebo }}>\mathrm{Wt}_{\text {initial }}$} \\
\hline Brainstem & & 8 & -14 & -18 & 3.79 & 956 \\
\hline Culmen & & -10 & -54 & -4 & 2.49 & 72 \\
\hline Globus pallidus & & -18 & -10 & 0 & 2.62 & 151 \\
\hline Inferior frontal gyrus & 47 & -46 & 28 & -4 & 3.09 & 339 \\
\hline Lingual gyrus & 18 & 18 & -84 & -4 & 2.77 & 431 \\
\hline \multirow[t]{2}{*}{ Middle frontal gyrus } & 9 & 38 & 22 & 34 & 3.21 & 1,070 \\
\hline & 9 & -52 & 22 & 38 & 3.16 & 477 \\
\hline Middle temporal gyrus & 21 & 58 & -2 & -18 & 2.84 & 94 \\
\hline \multirow{2}{*}{ Parahippocampal gyrus } & & -32 & -24 & -12 & 2.68 & 35 \\
\hline & & 32 & -26 & -12 & 2.54 & 62 \\
\hline \multicolumn{7}{|l|}{$\mathrm{Wt}_{\text {initial }}>\mathrm{Wt}_{-10 \% \text { placebo }}$} \\
\hline Amygdala & & -20 & -4 & -18 & 2.83 & 205 \\
\hline \multirow[t]{3}{*}{ Anterior cingulate gyrus } & 25 & -6 & 20 & -4 & 2.48 & 59 \\
\hline & 32 & -22 & 38 & 2 & 3.31 & 168 \\
\hline & 24 & 6 & 26 & 2 & 2.76 & 148 \\
\hline \multicolumn{7}{|l|}{ Cingulate gyrus } \\
\hline & 24 & 10 & -14 & 34 & 3.15 & 304 \\
\hline & 23 & -6 & -24 & 34 & 1.77 & 15 \\
\hline Fusiform gyrus & 37 & -48 & -36 & -18 & 2.66 & 78 \\
\hline Hypothalamus & & 12 & 6 & -12 & 2.19 & 60 \\
\hline Inferior parietal lobule & 40 & -40 & -58 & 40 & 1.86 & 12 \\
\hline Middle frontal gyrus & 11 & -24 & 50 & -12 & 2.00 & 14 \\
\hline Parahippocampal gyrus & 30 & -26 & -48 & 2 & 2.18 & 269 \\
\hline \multirow[t]{2}{*}{ Precentral gyrus } & 9 & 36 & -2 & 34 & 2.39 & 44 \\
\hline & 6 & -36 & -10 & 36 & 2.66 & 456 \\
\hline Supramarginal gyrus & 39 & 40 & -56 & 34 & 2.03 & 51 \\
\hline
\end{tabular}

Coordinates of peak cluster activity from the normalized brain were based on the Montreal Neurological Institute (MNI) system. Negative $x$ axis coordinates indicate left hemisphere. " $n$ voxels" indicates the number of voxels $\left(2 \mathrm{~mm}^{2}\right)$ in each cluster of activity. BA, Brodmann area.

A major finding of this study is that weight loss- and leptin-mediated effects on neural activity in response to food-related cues are observed in areas of the brain involved in both the regulation of feeding behavior and high-level executive processes. The similarities between subjects in euleptinemic states during the maintenance of usual weight and during leptin repletion following weight loss (Table 4) suggest a pivotal role for leptin in central mediation of the hunger associated with maintenance of reduced weight. Known physiological consequences of weight loss and leptin replacement are mirrored by congruent changes in PMRI activity in brain areas previously implicated in the context of such physiological phenotypes. Specifically, hypothalamic activity and activity in areas of the brain involved in emotional (cingulate gyrus; Brodmann area [BA] 24, 32) and cognitive (middle frontal gyrus) control are commonly engaged in states of leptin sufficiency and leptin repletion $(9,11-13)$. The pivotal role for leptin in the CNS-mediated responses to the maintenance of reduced weight is also demonstrated by the neural activity observed during the state of relative hypoleptinemia following weight loss relative to subjects at usual weight and leptin levels (Table 4). The regions include insula, cingulate gyrus, medial frontal gyrus, precuneus, middle occipital gyrus, superior frontal gyrus, and superior temporal gyrus (Table 2), suggesting that functions such as enteric regulation, emotional control, decision, and executive functions as well as high-

superior frontal gyrus) and high-level visual perception (middle occipital gyrus, lingual gyrus, and precuneus) functions (Figure 2D and Table 3). As hypothesized, some of these areas were similar to those that were more responsive during maintenance of a reduced body weight compared with $\mathrm{Wt}_{\text {initial }}$ (Figure $2 \mathrm{~A}$ and Table 2). These common areas included brainstem, culmen, parahippocampal gyrus, inferior and middle frontal gyri, middle temporal gyrus, and lingual gyrus.

\section{Discussion}

All results represent BOLD responses to the food items that exceeded those to the non-food items and can be considered food-related signals (11). Four pair-wise comparisons were made. Two isolated the effects of the maintenance of a reduced body weight on neural activity $\left(\mathrm{Wt}_{\text {initial }}>\mathrm{Wt}_{-10 \% \text { placebo }}\right.$ and $\left.\mathrm{Wt}_{-10 \% \text { placebo }}>\mathrm{Wt}_{\text {initial }}\right)$, and 2 isolated the effects of leptin repletion in a weight-reduced state $\left(\mathrm{Wt}_{-10 \% \text { leptin }}>\mathrm{Wt}_{-10 \% \text { placebo }}\right.$ and $\left.\mathrm{Wt}_{-10 \% \text { placebo }}>\mathrm{Wt}_{-10 \% \text { leptin }}\right)$. Each of these comparisons elicited a distinct pattern of neural activity representing a complex of neural responses during the viewing of food-related items. The distinct patterns of neural activity elicited in response to the same food cues under various physiological states related to changes in body energy stores and leptin provide information regarding models of CNS mechanisms associated with the regulation of body weight (Table 4). level perceptual systems are influenced by the relative reduction of leptin in the weight-reduced state.

Individuals maintaining a reduced body weight and humans or rodents that are congenitally leptin deficient or leptin unresponsive demonstrate similar behavioral (food seeking) and metabolic (reduced energy expenditure) phenotypes (14). Increased neural activity in the caudate nucleus, insula, and the frontal, parahippocampal, fusiform, and temporal gyri have been reported in PET scans of humans following food deprivation $(15,16)$. Our identification of leptin-specific effects of weight loss on metabolic activity of brain regions mediating food intake is consistent with earlier studies of the effects of weight loss and/or leptin administration on feeding behavior (12, 17-20) and energy expenditure (6) in animals and humans.

We hypothesized that the weight-stable, weight-reduced state is interpreted by neuronal systems regulating energy intake as a state of relative leptin deficiency (6). Farooqi et al. (17) reported that leptin administration to subjects with congenital leptin deficiency reduced fMRI responses to visual food stimuli in the anterolateral ventral striatum (including the nucleus accumbens and caudate) and posterolateral ventral striatum (including the putamen and globus pallidus) in 2 subjects with congenital leptin deficiency studied before and after 7 days of treatment with exogenous recombinant methionyl human leptin. Similarly, we detected leptin-reversible increases in activity following weight 
Table 3

Effects of leptin replacement

\begin{tabular}{|c|c|c|c|c|c|c|}
\hline \multirow[t]{2}{*}{ Region } & \multirow[t]{2}{*}{ BA } & \multicolumn{3}{|c|}{ Coordinates } & \multirow{2}{*}{$\begin{array}{c}z \\
\text { score }\end{array}$} & \multirow{2}{*}{$\begin{array}{l}\text { Voxels } \\
(n)\end{array}$} \\
\hline & & $x$ & $y$ & $z$ & & \\
\hline \multicolumn{7}{|l|}{$\mathrm{Wt}_{-10 \% \text { leptin }}>\mathrm{Wt}_{-10 \% \text { placebo }}$} \\
\hline \multirow[t]{2}{*}{ Cingulate gyrus } & 24 & \pm 14 & 36 & 0 & 2.56 & 651 \\
\hline & 32 & -16 & 18 & 38 & 2.67 & 450 \\
\hline Culmen & & -6 & -46 & -14 & 2.01 & 8 \\
\hline \multirow[t]{2}{*}{ Hypothalamus } & & -22 & -2 & -12 & 1.78 & 9 \\
\hline & & 12 & 14 & -2 & 1.84 & 21 \\
\hline \multirow[t]{2}{*}{ Inferior frontal gyrus } & 47 & 52 & 30 & -6 & 1.86 & 7 \\
\hline & 9 & 36 & -4 & 34 & 2.58 & 245 \\
\hline Lingual gyrus & 19 & -34 & -52 & -2 & 2.27 & 34 \\
\hline Medial frontal gyrus & 6 & 6 & 26 & 40 & 2.34 & 450 \\
\hline \multirow{3}{*}{ Middle frontal gyrus } & 11 & 26 & 34 & -18 & 1.83 & 12 \\
\hline & 11 & -26 & 44 & -12 & 2.59 & 208 \\
\hline & 6 & -38 & 6 & 42 & 2.06 & 78 \\
\hline Middle temporal gyrus & 20 & -54 & -32 & -18 & 1.91 & 22 \\
\hline Postcentral gyrus & 2 & -48 & -28 & 44 & 2.06 & 41 \\
\hline Precuneus & 19 & 42 & -76 & 40 & 2.11 & 61 \\
\hline Putamen & & -12 & 6 & -10 & 1.69 & 5 \\
\hline Thalamus & & \pm 2 & 2 & 4 & 2.56 & 488 \\
\hline \multicolumn{7}{|l|}{$\mathrm{Wt}_{-10 \% \text { placebo }}>\mathrm{Wt}_{-10 \% \text { leptin }}$} \\
\hline Brainstem & & -6 & -14 & -20 & 1.98 & 16 \\
\hline \multirow[t]{2}{*}{ Cingulate gyrus } & 23 & -18 & -20 & 34 & 2.30 & 66 \\
\hline & 24 & 26 & -4 & 40 & 2.23 & 41 \\
\hline \multirow[t]{2}{*}{ Culmen } & & 12 & -44 & -22 & 2.53 & 133 \\
\hline & & -14 & -40 & -20 & 2.37 & 73 \\
\hline Inferior frontal gyrus & 13 & -26 & 18 & -8 & 2.29 & 22 \\
\hline Insula & 13 & -36 & 6 & -10 & 2.23 & 41 \\
\hline Lingual gyrus & 18 & 20 & -84 & -4 & 2.04 & 21 \\
\hline Medial frontal gyrus & 10 & -6 & 64 & 0 & 1.97 & 9 \\
\hline \multirow[t]{2}{*}{ Middle frontal gyrus } & 10 & -36 & 58 & -6 & 2.28 & 10 \\
\hline & 9 & -28 & 12 & 32 & 2.37 & 69 \\
\hline Middle occipital gyrus & 37 & 32 & -74 & 0 & 2.50 & 36 \\
\hline Middle temporal gyrus & 21 & 42 & -38 & 0 & 2.29 & 42 \\
\hline \multirow[t]{2}{*}{ Parahippocampal gyrus } & 37 & -42 & -26 & -18 & 2.61 & 407 \\
\hline & 36 & 38 & -30 & -16 & 3.30 & 121 \\
\hline \multirow[t]{2}{*}{ Precuneus } & 19 & 28 & -64 & 28 & 3.01 & 126 \\
\hline & 31 & -20 & -50 & 30 & 2.16 & 29 \\
\hline Superior frontal gyrus & 9 & -12 & 40 & 38 & 1.87 & 17 \\
\hline Superior temporal gyrus & 21 & -44 & -4 & -10 & 2.11 & 50 \\
\hline
\end{tabular}

content was greater in the leptin-deficient than the leptin-replete study periods. Subjects in our study were imaged only in the post-absorptive state while remaining on a fixed liquid formula diet that was isocaloric between $\mathrm{Wt}_{-10 \% \text { placebo }}$ and $\mathrm{Wt}_{-10 \% \text { leptin }}$. Stimulus design was different between the studies as well. The observations by Farooqi et al. were obtained from subjects following 7 days of leptin administration and did not contain a placebo administration arm. Our studies were performed in subjects after at least 3 weeks of leptin therapy and included a placebo injection control period. Farooqi et al. used a picture block stimulus of 5 foods or non-foods per block and studied regions of interest, while we used a block design consisting of the actual presentation of 12 foods or non-foods per block. The study of leptin-deficient subjects does not specify the normalized atlas coordinates of the structures identified as being leptin responsive. The caudate, putamen, and ventral striatum are nexi for multiple functions, and as discussed above, it is quite possible that different functional regions of the ventral striatum and putamen were detected by virtue of the different study designs used in this investigation and that of Farooqi et al. (17). Finally, it is possible, if not likely, that there are irreversible effects of the leptin-deficient prenatal and perinatal environments of congenitally leptin-deficient subjects on the structural development of neuronal feeding circuits in humans, as has been show in $L e p^{o b}$ mice and Zucker rats (21).

While the differences between the designs of these studies do not permit a direct comparison of results, it is clear that reduced ambient leptin, whether due to congenital leptin deficiency or reduced

loss in the globus pallidus. We found that neuronal activity in the putamen in response to food cues was greater following administration of leptin compared with placebo, and we did not detect any weight loss- or leptin-mediated effects on the anterolateral ventral striatum or the putamen.

The differences between these 2 studies of leptin administration may reflect a number of distinct differences in study design, stimuli used, and in the study populations (17). More specifically, Farooqi et al. reported data that were similar between the 2 subjects studied and that were correlated to behavioral studies using a region of interest analysis, whereas with the exception of the hypothalamus, for which we also utilized a region of interest approach, we used a more conservative whole-brain analysis in a larger subject population and report only statistically significant changes that were evident with the a priori selection of specific regions of interest. The congenitally leptin-deficient subjects were studied in the fed and fasted states and remained on ad libitum mixed diet whose caloric body fat resulting from weight loss, increases neural activity in an extensive complex of brain areas in response to food cues. The leptin responsiveness in both leptin-deficient subjects who were not weight reduced and in subjects who were relatively hypoleptinemic by virtue to weight reduction indicates that the altered neural activity in response to food cues detected in the present study and in studies of fasted humans $(15,16)$ are due to reduced ambient leptin rather than to weight loss per se.

At both $\mathrm{Wt}_{-10 \% \text { placebo }}$ and $\mathrm{Wt}_{-10 \% \text { leptin }}$ (compared with $\mathrm{Wt}_{\text {initial }}$ ), we detected increased neural activity in response to food cues in the brainstem and anterior cingulate gyrus and relatively less activity in response to food cues in the amygdala. These changes in neural activity - which were not significantly altered by administration of exogenous leptin - may reflect the effects of other peripheral signals such as insulin and/or ghrelin levels, both of which change during maintenance of a reduced weight (9). Alternatively, these changes that are not reversed by leptin may reflect subject accom- 
A

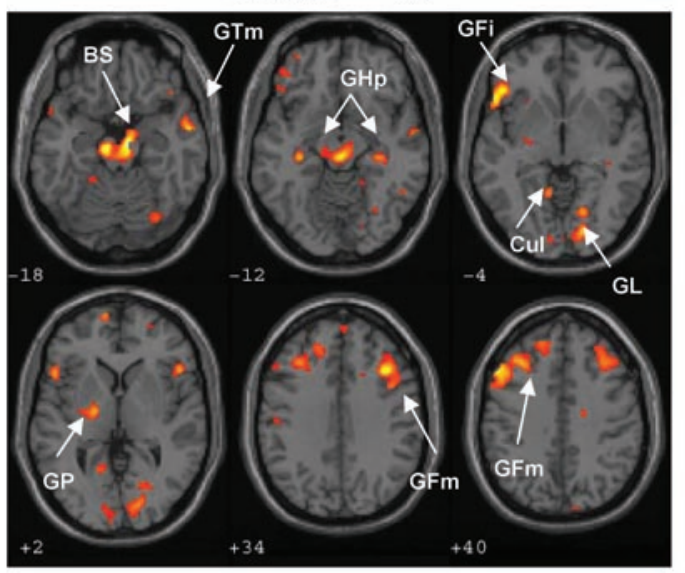

C

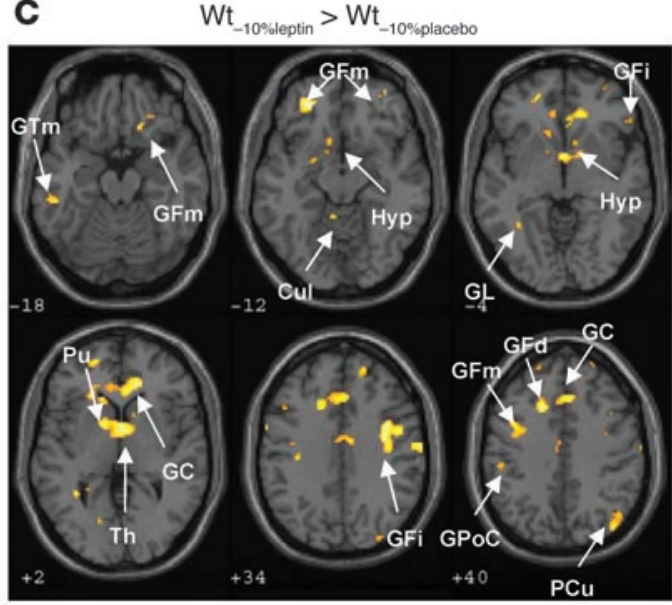

B

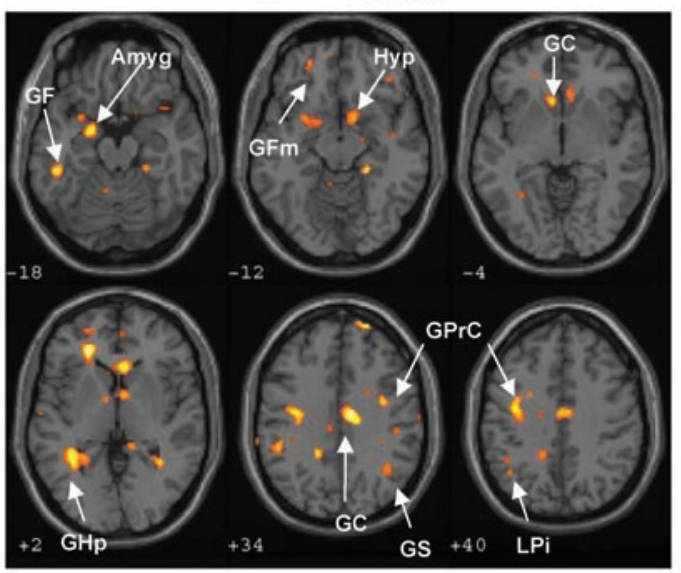

D

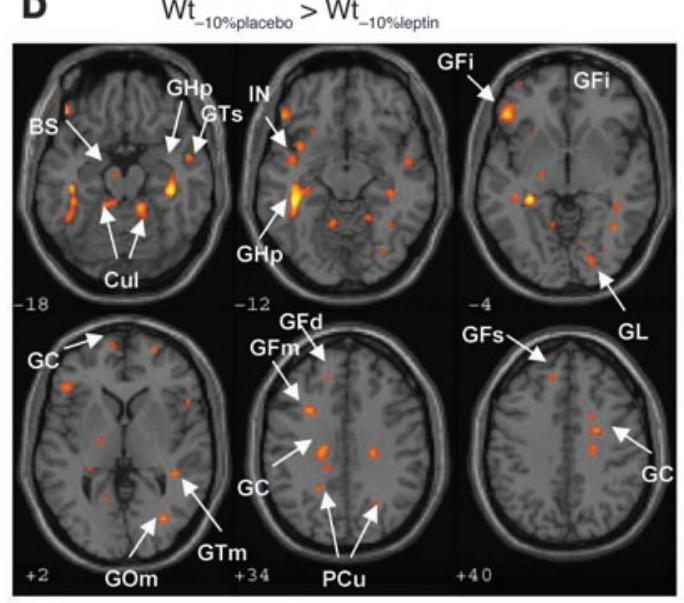

Figure 2

Effects of weight loss and leptin on neural activity following passive exposure to visual food cues. A and B show effects of weight loss. C and D show effects of leptin. Amyg, amygdala; BS, brainstem; Cul, culmen; GC, cingulate gyrus; GF, fusiform gyrus; GFm, middle frontal gyrus; GFs, superior frontal gyrus; GHp, parahippocampal gyrus; GOm, middle occipital gyrus; GP, globus pallidus; GPoC, postcentral gyrus; GPrC, precentral gyrus; GS, supramarginal gyrus; GTm, middle temporal gyrus; GTs, superior temporal gyrus; PCu, precuneus; Pu, putamen; Th, thalamus.

modation to repeated testing or subject reaction to the stress induced by receiving injections (placebo or leptin) at a reduced weight but not at $\mathrm{Wt}_{\text {initial }}$.

None of the brain activity patterns observed in this study were strongly lateralized to either hemisphere, although studies of taste have suggested left hemisphere bias (15). Many of the structures in which neural activation was detected by fMRI were medial and comprised of multiple tracts and nuclei, which may serve opposing functions. The hypothalamus is a good example of a multifunctional brain area. Ablative lesions of the lateral hypothalamus produce profound aphagia $(22,23)$; neural activity in this area would be predicted to increase following weight loss. In contrast, lesions of the region of the ventromedial hypothalamus produce the opposite phenotype, and activity in the ventromedial hypothalamus would be predicted to decrease following weight loss (22). We believe the present study to be unique in its ability to specifically identify neural activity changes in the hypothalamus using $\mathrm{AMRI}$, although the anatomic resolution $(1.5 \times 1.5 \times 4.5 \mathrm{~mm})$ is not sufficient to identify region-specific effects within the hypothalamus in a group study. The leptin-reversible changes in hypothalamic neural activity that occur following weight loss presumably reflect the net balance of neural activity in multiple neurons regulating energy intake.

As discussed above, changes in neural activity induced by weight loss and/or influenced by leptin repletion, in regions other than the hypothalamus, can be loosely grouped by function. There appear to be increases in activity during reduced-weight maintenance in systems relating to the emotional, executive, and sensory responses to food while there are decreases in systems involving emotional and cognitive control of food intake as well as integration of motor planning. Changes in neural activity in these areas following weight loss, mostly at the same loci and in all of the same systems, are reversed by leptin repletion. In addition, leptin repletion appears to increase activity in brain areas related to sensory discrimination and to diminish activity in areas sensing enteric signals and knowledge representation. Using this function-based analysis, changes in neural activity following weight loss would predict a phenotype of greater responsiveness to food (emotional and sensory responses to the presentation of food) with decreased control of food intake and discrimination based on quality or quantity of food consumed. 


\section{Table 4}

Overview of long-range patterns of brain activity observed in each of the comparisons relating the effects of weight maintenance, weight reduction, and leptin replacement

\begin{tabular}{|c|c|c|c|c|c|}
\hline \multirow[t]{2}{*}{ Anatomical region } & \multicolumn{2}{|c|}{ Weight loss effects } & \multicolumn{2}{|c|}{ Leptin effects } & \multirow[t]{2}{*}{ Putative function in energy intake } \\
\hline & $\begin{array}{c}\mathrm{Wt}_{-10 \% \text { placebo }} \\
>\mathrm{Wt}_{\text {initial }}\end{array}$ & $\begin{array}{c}W t_{\text {initial }}> \\
W t_{-10 \% \text { placebo }}\end{array}$ & $\begin{array}{l}\text { Wt }-10 \% \text { leptin }> \\
\text { Wt-10\%placebo }\end{array}$ & $\begin{array}{l}\mathrm{Wt}_{-10 \% \text { placebo }} \\
>\mathrm{Wt}_{-10 \% \text { leptin }}\end{array}$ & \\
\hline Amygdala & & $\mathrm{x}$ & & & $\begin{array}{c}\text { Regulates food intake in response } \\
\text { to sensory cues (33) }\end{array}$ \\
\hline Brainstem & $x$ & & & $x$ & $\begin{array}{l}\text { Processes stimulatory and inhibitory food } \\
\text { intake signals }\end{array}$ \\
\hline Cingulate gyrus & & $x$ & $x$ & $x$ & $\begin{array}{l}\text { Affects emotional self-control, problem solving, } \\
\text { and error recognition (dietary restraint) }(34)\end{array}$ \\
\hline Culmen & $\mathrm{x}$ & & $\mathrm{X}$ & $x$ & $\begin{array}{l}\text { Activity correlated with response-time inhibition (35), } \\
\text { creative thinking, working memory, and emotion (36) }\end{array}$ \\
\hline Fusiform gyrus & & $x$ & & & $\begin{array}{l}\text { Activity increases with fasting; influences food intake } \\
\text { in response to sensory stimuli (37) }\end{array}$ \\
\hline Globus pallidus & $x$ & & & & $\begin{array}{l}\text { Mediates the hedonic impact of } \\
\text { high-sugar/high-fat foods (10) }\end{array}$ \\
\hline Hypothalamus & & $x$ & $x$ & & Processes leptin and other humoral signals $(26,38)$ \\
\hline Insula & & & & $\mathrm{x}$ & $\begin{array}{l}\text { Involved in sensitivity to oral stimuli } \\
\text { related to feeding }(11,39)\end{array}$ \\
\hline Inferior frontal gyrus & $x$ & & $x$ & $x$ & $\begin{array}{l}\text { Processes food-related stimuli from the gut, } \\
\text { e.g., increases activity with gastric distention (40) }\end{array}$ \\
\hline Inferior parietal lobule & & $x$ & & & $\begin{array}{l}\text { Relates sensory data to past experience and } \\
\text { processes stimuli from gut }(40,41)\end{array}$ \\
\hline Lingual gyrus & $x$ & & $x$ & $x$ & $\begin{array}{l}\text { Relates affective response to high caloric } \\
\text { density foods (42) }\end{array}$ \\
\hline Medial frontal gyrus & & & $x$ & $x$ & Involved in object-reward decision making (43) \\
\hline Middle frontal gyrus & $x$ & $x$ & $x$ & $x$ & Relates sensory data to past experience (41) \\
\hline Middle occipital gyrus & & & & $x$ & $\begin{array}{l}\text { Less active in response to food cues in patients } \\
\text { with anorexia nervosa (13) }\end{array}$ \\
\hline Middle temporal gyrus & $x$ & & $x$ & $x$ & $\begin{array}{l}\text { Involved in recognition of visual stimuli and } \\
\text { decisions as to their function (44) }\end{array}$ \\
\hline Parahippocampal gyrus & $x$ & $x$ & & $x$ & $\begin{array}{l}\text { Mediates satiety and expresses leptin, CCK, } \\
\text { and cannabinoid receptors }(11,45,46)\end{array}$ \\
\hline Postcentral gyrus & & & $x$ & & $\begin{array}{l}\text { Involved in sensitivity to taste, smell, and } \\
\text { oromotor activity associated with eating (34) }\end{array}$ \\
\hline Precentral gyrus & & $x$ & & & $\begin{array}{l}\text { Involved in sensitivity to taste, smell, and } \\
\text { oromotor activity associated with eating (34) }\end{array}$ \\
\hline Precuneus & & & $x$ & $x$ & Relates sensory data to past experience (41) \\
\hline Putamen & & & $x$ & & $\begin{array}{l}\text { Greater activity in response to taste associated } \\
\text { with earlier satiety (47) }\end{array}$ \\
\hline Superior frontal gyrus & & & & $x$ & Relates sensory data to past experience (41) \\
\hline Superior temporal gyrus & & & & $x$ & $\begin{array}{l}\text { Activated in response to low caloric density foods } \\
\text { more than high caloric density foods (37) }\end{array}$ \\
\hline Supramarginal gyrus & & $x$ & & & Activity increases in response to pleasant taste (48) \\
\hline Thalamus & & & $x$ & & $\begin{array}{l}\text { More activity in response to visual presentation } \\
\text { of high vs. Iow caloric density foods (38) }\end{array}$ \\
\hline
\end{tabular}

The consequences of such leptin-reversible changes in the neural regulation of food intake in weight-reduced subjects are even more striking when viewed in the context of the effects of weight loss and leptin on energy output. We have previously shown that the maintenance of a $10 \%$ or greater reduced body weight is associated with leptin-reversible decreases in energy expenditure, sympathetic nervous system tone, and activity of the hypothalamic-pituitary- thyroid axis and leptin-reversible increases in the work efficiency of skeletal muscle $(6,14)$. The combination of decreased energy expenditure with a disinhibition of food intake in weight-reduced subjects would strongly promote the regain of lost weight. Current pharmacological weight loss therapies are based on the assumption of increased energy expenditure or decreased energy intake in individuals at usual body weight, i.e., when energy intake and 
output are balanced. However, weight loss promotes a dysregulation or uncoupling of this balance such that an increase in energy intake and a decrease in output support weight regain rather than weight maintenance. The leptin-reversible nature of this decline in energy output and increase in energy intake after weight loss suggests a possible role for pharmacological agents that stimulate the leptin-signaling pathway as enablers of reduced-weight maintenance rather than promoters of weight loss.

In summary, maintenance of a reduced body weight is accompanied by leptin-dependent changes in neural activity in regions of the brain implicated in vegetative/regulatory and hedonic aspects of energy homeostasis. We have previously shown that the maintenance of a reduced body weight is associated with leptin-reversible declines in energy expenditure and changes in neuroendocrine and autonomic function that mediate this decline in energy expenditure (6). We have also found that administration of leptin to weight-reduced subjects reverses the increased hunger and decreased satiety that characterize the weight-reduced state (24). Others have shown that hyperphagia is reversed by administration of leptin to congenitally leptin-deficient animals and humans (17, 25). Thus, low-leptin states provoke changes in energy intake and expenditure that act coordinately to favor the regain of lost body fat. The demonstration that the maintenance of a reduced body weight is associated with leptin-reversible changes in neural activity in brain areas known to mediate feeding-related behaviors further supports the pivotal role of leptin in body weight regulation as a primary "defense hormone" against loss of body fat following otherwise successful weight loss (26).

\section{Methods}

Subjects. Six obese (BMI, wt/height $\left.>30 \mathrm{~kg} / \mathrm{m}^{2}\right)(27)$ subjects ( 2 male, 4 female) were admitted to the General Clinical Research Center at Columbia University Medical Center and remained as inpatients throughout the study (see Table 1). All subjects had been stable at their maximal lifetime weights for at least 6 months prior to admission, were in good health, were taking no medications, and were right-hand dominant. Studies were approved by the Institutional Review Board of the New York Presbyterian Medical Center and were consistent with guiding principles for research involving humans subjects (28). Written informed consent was obtained from all subjects.

Protocol. See Figure 1 for a diagram of the protocol. Subjects were inpatients in the Clinical Research Center at Columbia Presbyterian Medical Center throughout this study. As described previously (29), subjects were fed a liquid formula diet ( $40 \%$ of calories as fat [corn oil], $45 \%$ as carbohydrate [glucose polymer], and $15 \%$ as protein [casein hydrolysate]), plus vitamin and mineral supplements, in quantities sufficient to maintain a stable weight (defined as an average daily weight variation of less than $10 \mathrm{~g} / \mathrm{d}$ for at least 2 weeks). This weight plateau was designated $\mathrm{Wt}_{\text {initial. }}$.

Following completion of the imaging studies at $\mathrm{Wt}_{\text {initial }}$ (described below), subjects were provided $800 \mathrm{kcal} / \mathrm{d}$ of the same liquid formula diet until they had lost $10 \%$ of $\mathrm{Wt}_{\text {initial. }}$. The duration of the weight loss phase ranged from 36 to 62 days. Once $10 \%$ weight loss had been achieved, caloric intake was adjusted upward until subjects were again weight stable. Subjects were then randomized to receive 5 weeks of twice-daily ( 8 am and $8 \mathrm{pm}$ ) s.c. injections of saline ( $\mathrm{Wt}_{-10 \% \text { placebo }}$ ) or 5 weeks of twice-daily ( 8 am and $8 \mathrm{pm}$ ) s.c. injections of recombinant methionyl human leptin (provided by Amylin Pharmaceuticals Inc.) in doses that were calculated to (and did) achieve circulating concentrations of leptin at 8 am (before injection) equal to those measured at $\mathrm{Wt}_{\text {initial }}(6)\left(\mathrm{Wt}_{-10 \% \text { leptin }}\right)$. Initial leptin doses were 0.08 and $0.14 \mathrm{mg} / \mathrm{kg}$ fat mass at $\mathrm{Wt}_{-10 \% \text { placebo }}$ for males and females, respec- tively (6). Circulating leptin concentrations at 8 am were measured weekly in subjects receiving leptin, and doses were adjusted until circulating leptin concentrations were similar to those measured at $8 \mathrm{am}$ at $\mathrm{Wt}_{\text {initial. }}$. Following completion of studies at $\mathrm{Wt}_{-10 \% \text { placebo }}$ or $\mathrm{Wt}_{\text {-10\%leptin, }}$ subjects underwent a 2-week washout period during which they received no injections. They were then crossed over to receive either leptin or placebo injections. Subjects were unaware of the order of testing and remained on a diet isocaloric (and identical in composition) to that initially shown to be sufficient to maintain a $10 \%$ reduced body weight throughout the leptin or placebo arms of the study.

Image acquisition. Images were acquired on a General Electric $1.5 \mathrm{~T}$ scanner equipped with $\mathrm{T} 2$-weighted echoplanar imaging sequences (time to repeat, $4,000 \mathrm{~ms}$; time to echo, $60 \mathrm{~ms}$; flip angle, $60^{\circ}$; field of view, $190 \times 190$ $\mathrm{mm}$; array size, $128 \times 128)(30)$. Twenty-five contiguous axial 4.5 -mm-thick slices were acquired parallel to the anterior-posterior commissure line with an in-plane resolution of $1.5 \times 1.5 \mathrm{~mm}$. Structural images were acquired with a T1-weighted spoiled gradient-recalled (SPGR) sequence (time to repeat, $19 \mathrm{~ms}$; time to echo, $5 \mathrm{~ms}$; flip angle, $20^{\circ}$; field of view, $220 \times 220$ $\mathrm{mm}$ ) recording 124 slices at a thickness of $1.5 \mathrm{~mm}$ with in-plane resolution of $0.86 \times 0.86 \mathrm{~mm}$. Although in some cases anatomical resolution can be improved at higher field strengths, the $1.5 \mathrm{~T}$ field strength was selected for this functional study because of the interest in the activity of ventral structures that are often compromised with higher field strength acquisitions due to air, bone, and soft tissue interfaces using the sequences necessary to detect the BOLD signal.

Image preprocessing. All preprocessing and statistical analyses were carried out using Statistical Parametric Mapping 2 (http://www.fil.ion.ucl.ac.uk./ $\mathrm{spm} / \mathrm{spm} 2 . \mathrm{html})$. Functional data were slice-time corrected and spatially realigned to the first volume of the first run. The structural scan was coregistered to the fMRI images and served to calculate transformation parameters for spatially warping fMRI images to the Montreal Neurological Institute template brain (resampled voxel size, $2 \mathrm{~mm}^{2}$ ). These normalized images were spatially smoothed with a $10-\mathrm{mm}^{3}$ kernel, and in order to remove the effects of low-frequency confounds, the data were high pass filtered at $128 \mathrm{~s}$. The first 3 volumes of each run were discarded prior to building and estimating the statistical models, as is standard practice in order to eliminate the effects of onset transients.

As previously described (11), subjects were tested in the postabsorptive state at 9 am and 72 images were acquired in each activation period, each of which lasted $4 \mathrm{~min} 48 \mathrm{~s}$. A block design was employed using 2 categories of visual stimuli: foods and non-foods. Each activation period consisted of 12 different foods or 12 different non-food items presented at 4-s intervals, and subjects underwent testing with 2 sets of food items and 2 sets of non-food items at each testing session. The order of activation periods was randomly assigned a priori but was retained at all testing sessions for each subject. Actual foods from multiple food groups (fruit, grains, vegetables, sweets) and actual non-foods (inanimate familiar objects such as a cell phone, jump rope, yo-yo) were placed in clear plastic containers and presented one at a time in view of the subject via a mirror on the head coil. Each item was viewed for 4 seconds, and subjects confirmed that all items were perceived following each run which consisted of 1 block and 2 baseline epochs. This design provided the advantage of feedback from the subject following each exposure epoch and is based upon routine mapping procedures established for localization of critical functions in preparation for neurosurgical procedures $(11,30)$.

Image and statistics. For each run, regressors for stimulus type (convolved with a canonical hemodynamic response function) were created for food and non-food stimuli and for $\mathrm{Wt}_{\text {initial, }}, \mathrm{Wt}_{-10 \% \text { placebo, }}$ and $\mathrm{Wt}_{-10 \% \text { leptin }}$ contrasts. Significant signal changes were identified with a voxel-by-voxel analysis based upon comparison of the mean signal amplitude during the periods 
of stimulation and the periods of resting baselines as determined by $t$ test comparisons using $P$ values of 0.005 corrected for multiple comparisons (11). This model was applied to each subject's data, followed by linear contrasts between conditions of interest, namely contrasting placebo, leptin, and initial weight activations. Contrasts were then compared in a random effects model across subjects based on a whole-brain analysis. Thresholds were constant at $P<0.05$ for units of 5 voxels. All regions that met statistical criteria were defined using the automatic labeling brain atlas (31) and applied with the Wake Forest University PickAtlas toolbox (http://fmri. wfubmc.edu/cms/software\#PickAtlas) (32).

Region-of-interest confirmation of the hypothalamus. As a regulatory locus for control of feeding behavior, the hypothalamus is a primary region of interest in this study. However, this small area is located at the interface of multiple tissue and media types (bone, air, and water), enhancing susceptibility to artifacts in fMRI images. Specifically, the hypothalamus is situated at the base of the skull just above the sella turcica, dorsal to the nasal sinus, and encapsulating the ventral portion of the third ventricle. Therefore, although salient signals in the region of the hypothalamus were consistently identified within the whole-brain analysis, a confirmation of these observations using a region-of-interest approach was performed. The structure of the region was identified independently of the functional signals using the PickAtlas (32). A morphologic dilatation factor of 3 was employed to define the masked area centered on the location of the hypothalamus region of interest. The coordinates of peak voxel clusters activated in regions attributed to the hypothalamus based on $\mathrm{AMRI}$ images (Figure $2 \mathrm{~B}, x, y, z=12,6,-12$; Figure 2C, $x, y, z=12,14,-2$ and $-22,-2,-12)$ fell

within the hypothalamus mask determined by anatomical features alone. Further, ROI analyses performed on the masked area revealed activity that coincided with the whole-brain approach (peak voxel, $x, y, z=8,4,-14$ and $6,0,-4)$. Thus, 2 sources of evidence converged to confirm the functional activity of the hypothalamus in this study, observed both in $\mathrm{Wt}_{\text {initial }}$ (Table 2) and $\mathrm{Wt}_{-10 \% \text { leptin }}$ (Table 3 ).

\section{Acknowledgments}

The authors acknowledge the invaluable contributions of the nursing and nutrition staff of the Clinical Research Center at Columbia University College of Physicians \& Surgeons/The New York Presbyterian Medical Center and also of the imaging staff and Stephen Dashnaw in the fMRI Research Center at Columbia University Medical Center. Recombinant methionyl human leptin was generously provided by Amylin Pharmaceuticals Inc. These studies were supported by NIH grants DK64473 and RR00645 and by grant UL1 RR0241576 from the National Center for Research Resources.

Received for publication January 17, 2008, and accepted in revised form April 23, 2008.

Address correspondence to: Michael Rosenbaum, Russ Berrie Medical Science Pavilion, Columbia University Medical College, Room 620, 1150 St. Nicholas Avenue, New York, New York 10032, USA. Phone: (212) 305-9949; Fax: (212) 851-5306; E-mail: mr475@columbia.edu.

1. Ogden, C., et al. 2006. Prevalence of overweight and obesity in the United States, 1999-2004. JAMA. 295:1549-1555.

2. Tsai, A., and Wadden, T. 2005. Systematic review: an evaluation of major commercial weight loss programs in the United States. Ann. Int. Med. 142:56-66.

3. Levin, B., and Keesey, R. 1998. Defense of differing body weight set points in diet-induced obese and resistant rats Am. J. Physiol. 274:R412-R419.

4. MacLean, P., et al. 2006. Peripheral metabolic responses to prolonged weight reduction that promote rapid, efficient regain in obesity-prone rats. Am. J. Physiol. 290:R1577-R1588.

5. Maclean, P., et al. 2004. Enhanced metabolic efficiency contributes to weight regain after weight loss in obesity-prone rats. Am. J. Physiol. 287:R1306-R1315.

6. Rosenbaum, M., et al. 2005. Low dose leptin reverses skeletal muscle, autonomic, and neuroendocrine adaptations to maintenance of reduced weight. J. Clin. Invest. 115:3579-3586.

7. Ahima, R., et al. 1996. Role of leptin in the neuroendocrine response to fasting. Nature. 382:250-252.

8. Weigle, D., Sande, K., Iverius, P., Monsen, E., and Brunzell, J. 1988. Weight loss leads to a marked decrease in nonresting energy expenditure in ambulatory human subjects. Metabolism. 37:930-936.

9. Gao, Q., and Horvath, T. 2007. Neurobiology of feeding behavior and energy expenditure. Ann. Rev. Neurosci. 30:367-398.

10. Kelley, A., Baldo, B., Pratt, W., and Will, M. 2005. Corticostriatal-hypothalamic circuitry and food motivation: integration of energy, action and reward. Physiol. Behav. 86:773-795.

11. St-Onge, M., Sy, M., Heymsfield, S., and Hirsch, J. 2005. Human cortical specialization for food: a functional magnetic resonance imaging investigation. J. Nutr. 135:1014-1018.

12. Hommel, J., et al. 2006. Leptin receptor signaling in midbrain dopamine neurons regulates feeding. Neuron. 51:678-680.

13. Santel, S., Baving, L., Kranel, K., Munte, T., and Rotte, M. 2006. Hunger and satiety in anorexia nervosa: $\mathrm{AMRI}$ during cognitive processing of food pictures. Brain Res. 1114:138-148.

14. Farooqi, I., et al. 2002. Beneficial effects of leptin on obesity, T cell hyporesponsiveness, and neuroendocrine/metabolic dysfunction of human congenital leptin deficiency. J. Clin. Invest. 110:1093-1103.

15. DelParigi, A., et al. 2002. Neuroimaging and obesity: Mapping the brain responses to hunger and satiation in humans using positron emission tomography. Ann. N. Y. Acad. Sci. 967:389-397.

16. Wang, G.J., et al. 2004. Exposure to appetitive food stimuli markedly activates the human brain. Neuroimage. 21:1790-1797.

17. Farooqi, I., et al. 2007. Leptin regulates striatal regions and human eating behavior. Science. 317:1355.

18. Bouret, S., Draper, S., and Simerly, R. 2004. Trophic action of leptin on hypothalamic neurons that regulate feeding. Science. 304:108-110.

19. Telles, M., Guimaraes, R., and Ribeiro, E. 2003. Effect of leptin on the acute feeding-induced hypothalamic serotonergic stimulation in normal rats. Regul. Pept. 115:11-18.

20. Williamson, D., et al. 2005. Microanalysis of eating behavior of three leptin deficient adults treated with leptin therapy. Appetite. 45:75-80.

21. Bouet, S., and Simerly, R. 2007. Development of leptin-sensitive circuits. J. Neuroendocrinol. 19:575-582.

22. Hetherington, A., and Ranson, S. 1942. The spontaneous activity and food intake of rats with hypothalamic lesions. Am. J. Physiol. 136:609-617.

23. Corbett, S., and Keesey, R. 1982. Energy balance of rats with lateral hypothalamic lesions. Am. J. Physiol. 242:E273-E279.

24. Kissileff, H., et al. 2007. Maintenance of reduced body weight in humans is associated with leptinreversible changes in appetite-related ratings [abstract]. Presented at the Society for Neuroscience Annual Meeting. November 3-7. San Diego, California, USA.

25. Schwartz, M. 2001. Brain pathways controlling food intake and body weight. Exp. Biol. Med. (Maywood). 226:978-981.
26. Leibel, R., Chua, S., and Rosenbaum, M. 2001. Obesity. In The metabolic and molecular bases of inherited disease. C. Scriver, A. Beaudet, W. Sly, and D. Valle, editors. McGraw-Hill. New York, New York, USA. 3965-4028.

27. Expert panel on the identification, evaluation, and treatment of overweight in adults. 1998. Clinical guidelines on the identification, evaluation, and treatment of overweight and obesity in adults: Executive Summary. Am. J. Clin. Nutr. 68:899-917.

28. American Physiological Society and World Medical Association General Assembly. 2002. Guiding principles for research involving animals and human beings. Am. J. Physiol. Regul. Integr. Comp. Physiol. 283:R281-R283.

29. Rosenbaum, M., et al. 1996. A comparative study of different means of assessing long-term energy expenditure in humans. Am. J. Physiol. 270:R496-R504.

30. Hirsch, J., et al. 2000. An integrated fmri procedure for preoperative mapping of cortical areas associated with tactile, motor, language, and visual functions. Neurosurgery. 47:711-722.

31. Tzourio-Mazoyer, N., et al. 2002. Automated anatomical labeling of activations in SPM using a macroscopic anatomical parcellation of the MNI MRI single-subject brain. NeuroImage. 15:273-289.

32. Maldjian, J., Laurienti, P., Kraft, R., and Burdette, J. 2003. An automated method for neuroanatomic and cytoarchitectonic atlas-based interrogation of fMRI data sets. Neuroimage. 19:1233-1239.

33. LaBar, K., et al. 2001. Hunger selectively modulates corticolimbic activation to food stimuli in humans. Behav. Neurosi. 115:493-500.

34. Allman, J., Hakeem, A., Erwin, J., Ninchinsky, E., and Hof, P. 2001. The anterior cingulate cortex: the evolution of an interface between emotion and cognition. Ann. N. Y. Acad. Sci. 935:107-117.

35. Simmonds, D., Fotedar, S., Suskauer, S., Pekar, J., Denckla, M., and Mostofsky, S. 2007. Functional brain correlates of response time variability in children. Neuropsychologia. 45:2147-2157.

36. Chavez-Eakle, R., et al. 2007. Cerebral blood flow associated with creative performance: a compara- 
tive study. Neuroimage. 38:519-528.

37. Killgore, W., and Yurgelun-Todd, D. 2005. Developmental changes in the functional brain responses of adolescents to images of high and low-calorie foods. Dev. Psychobiol. 47:377-397.

38. Killgore, W., et al. 2003. Cortical and limbic activation during viewing of high- versus low-calorie foods. Neuroimage. 19:1381-1394.

39. Augustine, J. 1996. Circuitry and functional aspects of the insular lobe in primates including humans. Brain Res. Brain Res. Rev. 22:229-244.

40. Stephan, E., et al. 2003. Functional neuroimaging of gastric distention. J. Gastrointest. Surg. 7:740-749.
41. Boisfueheneuc, F., et al. 2006. Functions of the left superior frontal gyrus in humans: a lesion study. Brain. 129:3315-3328.

42. Killgore, W., and Yurgelun-Todd, D. 2007. Positive affect modulates activity in the visual cortex to images of high calorie foods. Int. J. Neurosci. 117:643-653.

43. Walton, M., Bannerman, D., and Rushworth, M. 2002. The role of rat medial frontal cortex in decision making. J. Neurosci. 22:10996-1003.

44. Beauchamp, M., and Martin, A. 2007. Grounding object concepts in perception and action: evidence from fMRI studies of tools. Cortex. 43:461-468.
45. Iversen, P.O., and Nicolaysen, G. 1991. Local blood flow and glucose uptake within resting and exercising rabbit skeletal muscle. Am. J. Physiol. 260:H1795-H1801.

46. Harvey, J. 2003. Novel actions of leptin in the hippocampus. Ann. Med. 35:197-206.

47. Smeets, P., et al. 2005. Effect of satiety on brain activation during chocolate tasting in men and women. Am. J. Clin. Nutr. 83:1297-1305.

48. Gautier, J., et al. 1999. Regions of the human brain affected during a liquid-meal taste perception in the fasting state: a positron emission tomography study. Am. J. Clin. Nutr. 70:806-810. 